(pp 13-20)

\title{
Immediate Effects of Functional Electrical Stimulation on Kinematic and Kinetic Variables and Oxygenation in Medial Sensorimotor Cortices during Gait in Patients with Acute Cerebral Infarction
}

\author{
Masafumi KUBOTA, PT, PhD, Chiaki IGARASHI, PT, Hideaki MATSUO, PT, Hiroaki NARUSE, PT, \\ Seiichiro SHIMADA, PT \\ Division of Physical Therapy and Rehabilitation Medicine, University of Fukui Hospital \\ Osamu YAMAMURA, MD, PhD, Tomoko KAMISAWA, MD \\ Second Department of Internal Medicine, Faculty of Medical Sciences, University of Fukui \\ Ryu KATO, PhD, Hiroshi YOKOI, PhD \\ Department of Mechanical Engineering and Intelligent System, The University of Electro-Communications \\ Kenzo UCHIDA, MD, PhD, Hisatoshi BABA, MD, PhD \\ Department of Orthopaedics and Rehabilitation Medicine, Faculty of Medical Sciences, University of Fukui
}

Purpose: The aim of this study was to determine the immediate effects of functional electrical stimulation (FES) on kinematic and kinetic gait variables and oxygenation in medial sensorimotor cortices (mSMC).

Methods: We performed gait analysis using Vicon motion capture system and brain oxygenation in mSMC using near-infrared spectroscopy (NIRS) in eight subjects with acute cerebral infarction. FES was performed during walking on the tibialis anterior muscles and gastrocnemius of the paretic limb during the swing and terminal stance phases, respectively.

Results: Gait velocity, paretic hip flexion, knee extension, and ankle plantar flexion moments in the stance phase significantly increased when FES was performed during walking compared with those with walking before FES. Moreover, these improvements of gait quality were carried over after performing FES. Oxygenated hemoglobin levels in unaffected mSMC were significantly lower during walking with FES than that during walking without FES.

Conclusion: FES can improve weight shifting to the paretic limb during the stance phase and improve propulsion as a roll-off function in the late stance. Furthermore, FES may inhibit cortical excitability in unaffected mSMC. 